BULL. AUSTRAL. MATH. SOC.

$18 \mathrm{C} 15,18 \mathrm{~A} 35$

VOL. 36 (1987) 389-409

\title{
HOW COMPLETE ARE CATEGORIES OF ALGEBRAS?
}

\author{
JIŘÍ ADÁMEK
}

\begin{abstract}
Completeness properties of (i) the category Alg(T) of $T$-algebras over a functor $T: X \rightarrow X$ and (ii) the subcategory $x^{T}$ in the case where $T=(T, \mu, n)$ is a monad, are investigated. It is known that if $X$ is compact, then each $x^{\top}$ is compact; we present a functor $T:$ Set $\rightarrow$ Set such that Alg(T) is non-compact, although it is hypercomplete. If $T$ either preserves epis or has a rank, we prove that $A l g(T)$ and $X^{T}$ are topologically algebraic over $X$ provided $X$ satisfies mild additional hypotheses. Nevertheless, a natural monad over the category of $\Delta$-complete posets is exhibited such that its category of algebras is solid, but not topologically algebraic, over set.
\end{abstract}

\section{Introduction}

Two examples of categories of algebras form the background of the present paper. One is a hypercomplete category which is not compact, thus answering the problem as to whether these two properties are equivalent, put by Borger, Tholen, Wischnewsky and Wolff [6]. The other is a solid

Received 13 November 1986. I am sincerely grateful to Max Kelly Jan Reiterman, and Walter Tholen who helped me by catching a number of errors in my manuscript.

Copyright Clearance Centre, Inc. Serial-fee code: 0004-9727/87 $\$ A 2.00+0.00$. 
category of unary ordered algebras which is not topologically-algebraic ovex set, the first "natural" example of this sort.

By categories of algebras are meant the categories Alg(T) given by a functor $T: X \rightarrow X$ : objects are $T$-algebras, that is maps $T X \stackrel{x}{\longrightarrow} X$ (no axions), morphisms from $T X \stackrel{x}{\longrightarrow} X$ to $T Y \stackrel{y}{\longrightarrow} Y$ are maps $f: X \rightarrow Y$ in $X$ with $f \cdot x=y . T f$. The four properties mentioned above are studied for categories Alg(T) and, in the case where $T$ is the functor-part of a monad $T$, for their full subcategories $x^{\top}$. For the latter, compactness as well as hypercompleteness are lifted from $X$ to $X^{\top}$, see [15]. The same is, consequently, true for $A \lg (T)$ if $T$ is a varietor, that is, if each $X$-object generates a free $T$-algebra : then $\operatorname{Alg}(T)=X^{T^{*}}$ for the free monad $T^{*}$ generated by $T$, see [4]. The quotient $P^{*}$ of the covariant power-set functor obtained by merging finite sets has the property that $\mathrm{Alg}\left(P^{*}\right)$ is hypercomplete but noncompact. It is an open problem whether compactness of Alg(T) implies that $T$ is a varietor.

The concepts of solid (= semi-topological) and topologically-algebraic categories have both been created in order to express the common features of topological and algebraic concrete categories. But whereas each topological category is clearly topologically-algebraic (which is slightly stronger than solid, see [5] and [7]), the situation with algebraic categories is not that clear. For "reasonable" base categories we prove that $A \lg (T)$ and $X^{\top}$ are topologically-algebraic whenever $T$ either preserves epis, or has a rank. However, the non-cocomplete monadic category over graphs, presented in [1], is not solid (since solid functors lift colimits). We present a monad $T$ over the category $X$ of $\Delta$-complete posets such that $X^{\top}$, as a concrete category over set, is solid, but not topologically-algebraic. However, it is not known whether a monadic category $X^{\top}$ is topologically-algebraic (over $X$ ) whenever it is solid. 


\section{Compactness and Hypercompleteness}

1.1 Recall from [6] that a category $K$ is said to be hypercomplete if it has limits of all (possibly largel diagrams $D$ with the property that for each $K \in K$ the collection of all compatible sources of $D$ with the domain $K$ is small. (Since these sources are in a bijective correspondence with hom $(K, \lim D)$, this is the strongest completeness possible in a locally small category.)

1.2 A class $M$ of monos in a cocomplete category $X$ is said to be chain-cocomplete if for each $M$-chain the colimit maps are in $M$, and the factorization map of any compatible source of $M$-monos is also a $M$-mono.

1.3 PROPOSITION. Let $X$ be a cocomplete, hypercomplete, weilpowered category, and let $M$ be a chain-cocomplete class of monos containing all coproduct injections. Then $A \mathrm{Ig}(T)$ is hypercomplete for each functor $T: X \rightarrow X$ preserving $M-$ monos $(T M \subseteq M)$ and such that there is an object $X$ isomorphic to $T X+Y$ for some separator $Y$.

Proof. It is proved in [18] that the separator $Y$ generates a free $T$-algebra, that is, there is a $T$-algebra $K$ and a universal arrow $Y \rightarrow U K$ (where $U: \operatorname{Alg}(T) \rightarrow X$ denotes the forgetful functor). Let $D$ be a diagram of $T$-algebras satisfying the condition of 1.1 in Alg(T). Then the underlying diagram U.D satisfies the corresponding condition in Set:

(a) the compatible sources of U.D with the domain $Y$ form a small collection because they are in a bijective correspondence with the compatible sources of $D$ with domain $K$, and

(b) the compatible sources of U.D with any other fixed domain $Y^{\prime}$ form a small collection because $Y^{\prime}$ is a quotient of a co-power of $Y$. This proves that $\lim U . D$ exists in $X$, and since $U$ creates limits [14], $\lim D$ exists also.

COROLLARY. Alg(T) for a set functor $T$ is hypercomplete whenever there is a set $X \neq \emptyset$ with card $T X \leq$ card $X-1$. 
In fact, the hypotheses of the proposition are satisfied for $M$ = monos. Each set functor $T$ preseryes non-empty monos, and we can redefine $T$ on the empty set, putting $T^{\prime} X=T X$ for all $X \neq \emptyset$ and $T^{\prime} \emptyset=$ (and analogously on mapsl to obtain a set functor $T^{\prime}$ preserving all monos. The hypercompleteness of Alg(T) is clearly equivalent to that of $\operatorname{Alg}\left(T^{\prime}\right)$.

Open problem. If $\mathrm{Alg}(T)$ is hypercomplete for a set functor $T$, does it follow that there is a set $X \neq \emptyset$ with $\operatorname{card} T X \leq \operatorname{card} X-1$ ?

1.4 EXAMPLE. For the power-set functor $P[P X=\exp X$ and Pf : $M \rightarrow f(M)]$, the category $A l g(P)$ is not hypercomplete.

In fact, for each cardinal $n>0$ we can choose a map $x_{n}:$ Pn $\rightarrow n$ satisfying

$$
x_{n}(M) \notin M \text { for any set } M \varsubsetneqq n \text {. }
$$

Then the large discrete diagram of all of these algebras $\left(n, x_{n}\right)$ does not have a compatible source in $A l g(P)$. In fact, consider an arbitrary $P$-algebra $(Y, y)$. Given a homomorphism $f:(Y, y) \rightarrow\left(n, x_{n}\right)$ for some cardinal $n$, then

$$
x_{n}(P f P(Y))=f \cdot y(Y) \in P f(Y)
$$

which implies $P f(Y)=n$, that is, $f$ is onto. Thus, card $Y \leq n$.

On the other hand, in a hypercomplete category each diagram obviously has a compatible source.

1.5 Recall from [11] that a category $K$ is said to be compact if each functor with domain $K$ preserving all (possibly large) colimits existing in $K$ is a left adjoint.

1.6 PROPOSITION. $A g\left(P^{*}\right)$ is hypercomplete but non-compact for the quotient $P^{*}$ of the power-set functor modulo finite sets, that is, the following functor

$$
P^{*} X=\{M \mid M \subseteq X \text { and } M \text { is infinite, or } M=\emptyset\}
$$


and

$P^{*} f(M)=P f(M)$ if $P f(M)$ is infinite, else, $P^{*} f(M)=\emptyset$.

Proof. Alg $\left(P^{*}\right)$ is hypercomplete by 1.3 , since for a two-point set $X$ we have $\operatorname{card} P^{*} X=1=\operatorname{card} X-1$.

Let $\tilde{P}$ denote the quotient of the power-set functor modulo singletons, that is, $\tilde{P} X=\{M \mid M \subseteq X$ and card $M \neq 1\}$ and $\tilde{P} f(M)=P f(M)$ if card $P f(M) \neq 1$, else $\tilde{P} f(M)=\emptyset$. We shall prove that the natural full embedding

$$
E: \operatorname{Alg}\left(P^{*}\right) \rightarrow \operatorname{Alg}(\tilde{P})
$$

assigning to each. $P^{*}$-algebra $P^{*} X \stackrel{x}{\longrightarrow} X$ the corresponding $\tilde{P}$-algebra $\tilde{P} X \stackrel{\varepsilon}{\longrightarrow} R^{*} X \stackrel{x}{\longrightarrow} X \quad$ (for the obvious epitransformation $\varepsilon: \tilde{P} \rightarrow P^{*}$ ) preseryes colimits, but is not a left adjoint. The lattex is obvious : for $X=\{0,1,2\}$ define $x: \tilde{P} X \rightarrow X$ by $x(M)=0$ for all $M \neq X$ and $x(X)=1$. Then the $\tilde{P}$-algebra $(X, x)$ does not have a co-universal arrow from $E$ because both $\{0,1\}$ and $\{0,2\}$ are subalgebras lying in $E\left(A \lg \left(P^{*}\right)\right)$ but the algebra $(X, x)$ itself does not lie there.

To prove that $E$ preserves colimits, we can restrict ourselves to non-empty diagrams $D: D \rightarrow A l g\left(P^{*}\right)$, since $E$ clearly preserves initial $\Leftrightarrow$ singletonl algebras. We prove the following statement :

(*) If $D$ has a colimit $e_{d}: D d=\left(x_{d}, x_{d}\right) \rightarrow(y, y), d \in D^{p b j}$ then there exists $d_{0}$ such that $e_{d_{0}}$ is onto.

Once (*) is established, we shall see that the diagram $E . D$ has the colimit $e_{d}:\left(X_{d}, x_{d} \cdot \varepsilon_{X_{d}}\right)+\left(Y, y, \varepsilon_{Y}\right)$ as follows. Let

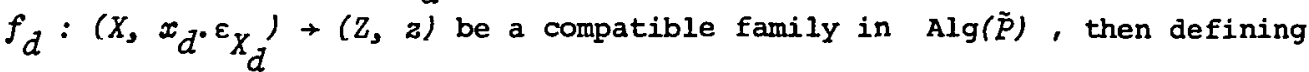
$\bar{z}: P^{\star} Z+Z$ by $\bar{z}(M)=z(M)$ for all $M \in P^{\star} Z$, we have clearly a compatible family $f_{d}:\left(X_{d}, x_{d}\right) \rightarrow(z, \bar{z})$ in $A l g\left(p^{*}\right)$. There is a unique $P^{*}$-homomorphism $f:(Y, y) \rightarrow(z, \bar{z})$ with $f_{d}=f \cdot e_{d}$, and it is sufficient to show that $f:\left(y, y, \varepsilon_{Y}\right)(Z, z)$ is a $\tilde{P}$-homomorphism. For 
each $M \in \tilde{P} Y$ we are to show that $z \cdot P f(M)=f \cdot y \cdot \varepsilon_{Y}(M)$. This is clear if $f(M)$ is infinite, since then $\tilde{p} f(M)=P^{*} f(M)$. For $f(M)$ finite there exists $M^{\prime} \subseteq X_{d_{0}}$ finite with $f(M)=f_{d_{0}}\left(M^{\prime}\right)$, see $(*)$, and hence,

$$
z \cdot \tilde{P} f(M)=z \cdot \tilde{P} f_{d_{0}}\left(M^{\prime}\right)=f_{d_{0}} \cdot x_{d_{0}} \cdot \varepsilon_{X_{d_{0}}}\left(M^{\prime}\right)=f_{d_{0}} \cdot x_{d_{0}}(\not)
$$

because $f_{d_{0}}$ is a $\tilde{p}$-homomorphism. Since $e_{d_{0}}$ is a $\tilde{p}$-homomorphism, we have $e_{d_{0}} \cdot x_{d_{0}}(\phi)=y(\phi)$ and thus,

$$
z \cdot \tilde{P} f(M)=f \cdot e_{d_{0}} \cdot x_{d_{0}}(\emptyset)=f \cdot y(\emptyset)=f \cdot y \cdot \varepsilon_{Y}(M) .
$$

In order to proye (*), we assume the contrary, and derive a contradiction. Let $n$ be a cardinal larger than card $Y$ and assume, for simplicity, that $Y \cap n=\emptyset$, that is, $Y$ does not contain ordinals $i<n$. Define a $P^{*}$-algebra $(Y \cup n, \bar{y})$ by

$$
\bar{y}(M)= \begin{cases}y(M) & \text { for all } M \in P^{*} X, M \neq Y \\ i & \text { for } M=Y \cup i, i<\eta \\ 0 & \text { otherwise }\end{cases}
$$

Denote by $e_{d}^{\prime}: X_{d} \rightarrow Y \cup n$ the range-extension of $e_{d}$, then $e_{d}^{\prime}:\left(X_{d}, x_{d}\right) \rightarrow(Y \cup n, \bar{y})$ is a compatible family of the given diagram : for each $M \in P^{*} X_{d}$ we have $P^{*} e_{d}^{\prime}(M) \neq Y$ (else, $e_{d}$ would be onto, contrary to our assumption) and hence, $\bar{y} \cdot P^{*} e_{d}^{\prime}(M)=y \cdot P e_{d}(M)$, which proves

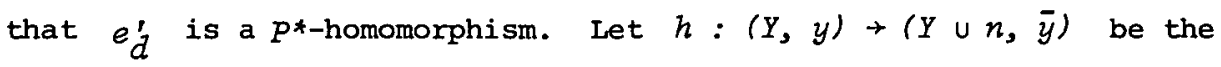
unique $P^{*}$-homomorphism with $h_{\cdot} e_{d}=e_{d}^{\prime}$, that is, with

$$
h(x)=x \text { for all } x \in \cup e_{d}\left(X_{d}\right)
$$

We shall prove by induction on $i<n$ that $Y u i \subseteq h(Y)$; this clearly contradicts card $h(Y) \leq \operatorname{card} Y<n$.

First step: $Y \subseteq h(Y)$. The set $Y_{0}=Y \cap h(Y)$ is a subalgebra of $(y, y)$. In fact, for each $M \in P^{*} Y_{0}$ there is $N \in P^{*} Y$ with 
$M=P^{*} f(N)$, and we can suppose that $\bar{y}(M)=y(M)$ (for otherwise $M=Y$ which implies $Y \subseteq h(Y)$ anyhowl. Thus,

$$
y(M)=\bar{y} \cdot P^{*} f(N)=h \cdot y(N) \in h(Y),
$$

and this implies $y(M) \in Y_{0}$. The subalgebra $Y_{0}$ contains $U e_{d}\left(X_{d}\right)$ and hence, we can form range-restrictions of the $P^{*}$-homomorphisms $e_{d}$ to $y_{0}$. This clearly implies that $y_{0}=Y$, that is, that $Y \subseteq h(Y)$.

At a successor ordinal we show that $Y \cup i \subseteq h(Y)$ implies $i \in h(Y)$ [and thus, $Y \cup(i+1) \subseteq h(Y)]$. Let $M \in P^{*} Y$ be a set with $P h(M)=y \cup i$ then

$$
i=\bar{y} \cdot P^{*} h(M)=h \cdot y(M) \in h(Y) .
$$

The limit steps are clear.

\section{Topological-algebraicity and solidness}

2.1 A concrete category $K$ over a base-category $X$ is said to be topologically-algebraic if it has free objects and (epi, initial)factorization of sources. This concept, introduced by Y.H. Hong [10] and S.S. Hong [9], aims to express the comon features of topological and algebraic categories. The same is the aim of the following, somewhat weaker, property : $K$ is said to be solid if each structured sink $\left|K_{i}\right| \stackrel{f_{i}}{\longrightarrow} X(i \in I), K_{i} \in K$, has a semifinal $1 i f t$, that is an object $K \in K$ and a morphism $n: X \rightarrow|K|$ such that

(a) all n. $f_{i}: K_{i} \rightarrow K$ are $K$-morphisms and

(b) if $h: X \rightarrow|L|$ is a map such that all $h \cdot f_{i}: K_{i} \rightarrow L$ are $K$-morphisms, then there is unique $h^{\#}: K \rightarrow L$ in $K$ with $h=h^{\#} \cdot n$. The concept of a solid category was introduced by [8], [16] and [17], and has often been called a semi-topological category. The following has been proved in [5] and [7] for each cocomplete, cowellpowered base-category $X$ : (i) Topologically-algebraic $\Rightarrow$ solid $\Rightarrow$ cocomplete and has free objects; and 
(ii) The reverse implications hold whenever the concrete category $K$ is cowellpowered.

Moreover, each reflective full subcategory of a solid category is solid, and solid forgetful functors are closed under composition (and they form the composition hull of topologically-algebraic forgetful functors).

\subsection{LEMMA. In each monadic concrete category, monosources are} initial.

Proof. Let $m_{i}:(X, x) \rightarrow\left(Y_{i}, y_{i}\right), i \in I$, be a monosource in $X^{\top}$. Then $m_{i}: X+Y_{i}(i \rightarrow I)$ is a monosource in $X$. If $p, q: Z \rightarrow X$ fulfil $m_{i} \cdot p=m_{i} \cdot q$, then for the homomorphisms $x . T p, x . T q:\left(T Z, \mu_{z}\right) \rightarrow(X, x)$ we have

$$
m_{i} \cdot x \cdot T p=y_{i} \cdot T\left(m_{i}, p\right)=y_{i} \cdot T\left(m_{i}, q\right)=m_{i} \cdot x \cdot T q,
$$

and hence $x \cdot T p=x \cdot T q \cdot$ Consequently

$$
p=x \cdot \eta_{X} \cdot p=x \cdot T p \cdot \eta_{Z}=x \cdot T q \cdot \eta_{Z}=q .
$$

Let $(Z, z)$ be a T-algebra, and let $h: Z \rightarrow X$ be a map such that each $m_{i}, h$ is a homomorphism, that is,

$$
m_{i} \cdot h \cdot z=y_{i} \cdot T m_{i} . T h=m_{i} \cdot x . T h \quad(\text { for all } i \text { ). }
$$

Then $h . z=x . T h$, that is, $h$ is a homomarphism.

2.3 PROPOSITION. Let $x$ be a complete category with intersections. Then each monadic category over $X$ which is cowellpowered with respect to extremal epis is topologically-algebraic.

Proof. By the preceding Lemma, it is sufficient to show that each category $x^{\top}$, cowellpowered with respect to extremal epis, has (epi, monosource)-factorizations of sources.

Since monadic functors create (large) limits, see [14], the category $x^{\top}$ is complete and has intersections. Consequently, it has (extremal epi, 
monol-factorizations of morphisms; it follows immediately that each small source $f_{i}: A \rightarrow A_{i}(i \in I)$ has an (extremal epi, monosource)-factorization:. factor the corresponding morphism $A+\prod_{i \in I} A_{i}$. Finally, for

large sources, we factor a small subsource representing all of the extremal co-images of the members of the source.

EXAMPLE. There is a monad $T$ over Pos, the category of posets, such that Pos $^{\top}$ is not topologically-algebraic. In fact, a monad $T$ such that Pos $^{\top}$ is not cocomplete is exhibited in [3], and topologicallyalgebraic functors lift colimits.

\subsection{PROPOSITION. Let $x$ be a cocomplete, E-cowellpowered} category with a morphism factorization system $(E, M)$. For each functor $T: X+X$ preserving E-epis (that $i s, T E \subseteq E$ ), equivalent are:

(i) $T$ is a varietor;

(ii) $A \lg (T)$ is topologically-algebraic.

Remark. Furthermore, for each monad $T$ preserving E-epis, $x^{\top}$ is topologically-algebraic - the proof is analogous.

Proof. $i i+i$ is clear since a topologically-algebraic category always has free objects. To prove $i \rightarrow i i$, it is sufficient to show that if $T$ is a varietor then $A l g(T)$ has ( $E$, monosource)-factorization of source - since $E \subseteq$ Epi and each monosource is initial, this will prove that $\mathrm{Alg}(T)$ is topologically-algebraic.

It is proved in [3] that $A l g(T)$ is cocomplete. It has (E, $M)$ factorizations of morphisms because for each homomorphism $f:(X, x)+(Y, y)$ with $(E, M)$-factorization $f=m . e$ in $x$, the diagonal fill-in yields an operation map such that $m$ and $e$ become homomorphisms : 


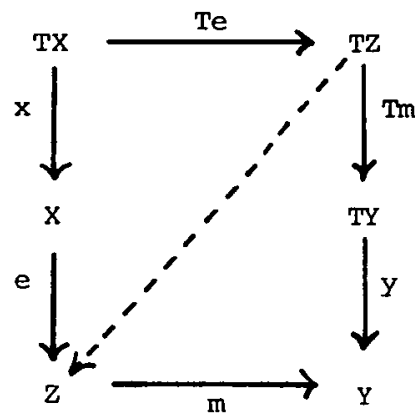

For each source $f_{i}: A \rightarrow B_{i}(i \in I)$ let $e: A \rightarrow A^{\prime}$ be the cointersection of all E-quotients of $A$ in $A l g(T)$ through which each $f_{i}$ factors, then $e \in E$ and we have factorizations $f_{i}=f_{i}^{\prime} . e(i \in I)$. The source $f_{i}^{\prime}: A^{\prime}+B_{i}(i \in I)$ is a monosource : if $p, q: C+A^{\prime}$ are merged by each $f_{i}^{\prime}$, then each $f_{i}^{\prime}$ factors through the coequalizer $c$ of $p$ and in $\operatorname{Alg}(T)$, and hence, each $f_{i}$ factors through c.e EE-consequently, c is an isomorphism.

2.5 DEFINITION. Let $X$ be a category with a morphism factorization system (E, M) and with colimits of M-chains.

A functor $T: X \rightarrow X$ is said to have rank $k$, where $k$ is a regular cardinal, if for each chain $V: k+X$ of $M$-monos the canonical morphism colim $(T . V) \rightarrow T(\operatorname{colim} V)$ is an $E$-epi.

2.6 Remark.. It is often the case that the class $M$ of all monos is chain-cocomplete (see 1.2), and that rank $k$ implies that colimits of $k$-chains of $M$-monos are actually preserved (for example, this holds for mono-preserving functors on $X=$ Set, Top, Pos, varieties of finitary algebras. .

For functors $T$ with rank, the category Alg(T) was proved to be solid by Koubek and Reiterman [13] (assuming $X$ cocomplete and E-cowellpowered). Using the technique developed by Barr [4], we are able to prove more: 
2.7 THEOREM. Let $x$ be a cocomplete, E-cowellpowered category with a factorization system $(E, H)$ ouch that $M$ is chain-cocomplete. Then $A \lg (T)$ is topologically-algebraic for each functor $T: X \rightarrow X$ with rank.

Remark. Furthermore, for each monad $T$ with rank, $x^{T}$ is topologically-algebraic. The proof is analogous.

Proof. It has been proved by Koubek and Reiterman in [13] that $T$ is a varietor and $\mathrm{AIg}(\mathrm{T})$ is cocomplete.

Thus, it is sufficient to prove that $\mathrm{Alg}(T)$ is cowellpowered with respect to extremal epis. Then $\mathrm{Alg}(T)$ has (extremal epi, monosource)factorization of sources : for each source $f_{i}: A \rightarrow B_{i}(i \in I)$ let $e: A \rightarrow A^{\prime}$ denote the cointersection of all extremal quotients of $A$ in Alg(T) through which each $f_{i}$ factors $(i \in I)$. Then each $f_{i}$ factors as $f_{i}=f_{i}^{\prime} \cdot e$, and the source $f_{i}^{\prime}: A^{\prime} \rightarrow B_{i}(i \in I)$ is clearly a monosource. Since $T$ is a varietor, monosources are initial in Alg(T) by 2.2, and this will establish $\mathrm{Alg}(T)$ as a topologically-algebraic category.

Let $k$ denote the rank of $T$. For each object $X$ of $X$ define a small set $x^{*}=\underset{i<k}{\cup} M_{i}$ of objects inductively as follows:

$$
\begin{aligned}
& M_{0}=\{X\} \\
& M_{i+1}=\left\{\begin{array}{l}
\text { closure of } M_{i} \text { under T-images and } \\
\text { colimits of diagrams with less than } \\
k \text { objects ( } i \text { even): } \\
\text { a choice set of all E-quotients of } \\
\text { all } M_{i} \text {-objects }(i \text { odd), }
\end{array}\right. \\
& M_{i}=\underset{j<1}{\cup} M_{j} \text { for limit ordinals } i .
\end{aligned}
$$

The proof will be concluded if we verify that for each extremal epi $f:(X, x) \rightarrow(Y, y)$ in Alg(T), $Y$ is isomorphic to an object in $X^{*}$ : since $X^{*}$ is a small set, it follows that Alg(T) is cowellpowered with respect to extremal epis. 
Define a chain $V: k+1 \rightarrow X$ and a natural $M$-transformation $m_{i}: V_{i}+Y(i \leq k)$ by the following induction:

(i) factor $f$ as $e_{0}: X \rightarrow V_{0}$ in $E$ followed by $m_{0}: V_{0} \rightarrow Y$ in $M$; (ii) given $m_{i}: V_{i} \rightarrow Y$, factor the morphism $\left(m_{i}, y . T m_{i}\right): V_{i}+T V_{i} \rightarrow Y$ as an E-epi (having compoents $V_{i, i+1}: V_{i} \rightarrow V_{i+1}$ and $d_{i}: T V_{i} \rightarrow V_{i+1}$ '

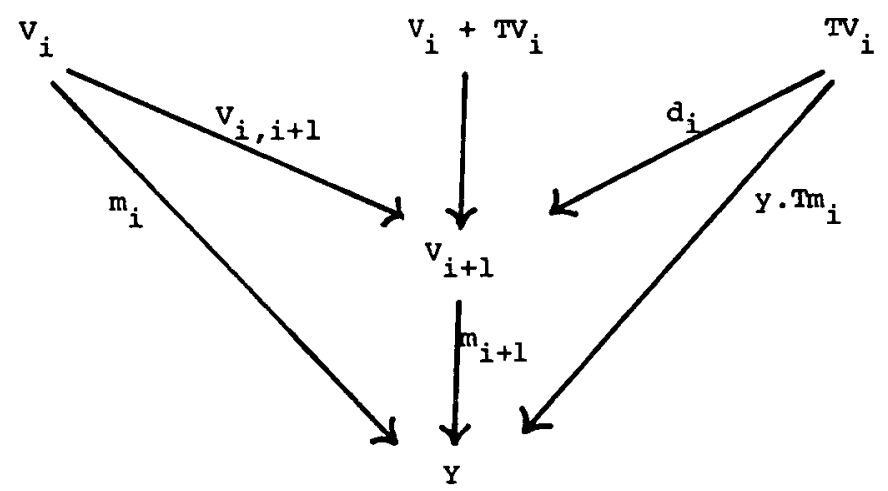

followed by $m_{i+1}: V_{i+1} \rightarrow Y$ in $M$;

(iii) for each limit ordinal $i, V_{i}=\underset{j<i}{\operatorname{colim}} V_{j}$ and $m_{i}=\underset{j<i}{\operatorname{colim}} m_{j}$ (which is in $M$ since $M$ is chain-cocomplete).

Observe that $V_{i} \in X^{*}$ for each $i \leq k$. Since $T$ has rank $k$, the canonical morphism

$$
c: \underset{i<k}{\operatorname{colim}} T V_{i} \rightarrow T V_{k}
$$

is an E-epi. Let $d: \operatorname{colim} T V_{i} \rightarrow V_{k}$ be the colimit map of the morphisms $d_{i}: T V_{i} \rightarrow V_{i+1}(i<k)$, then the square below commutes. In fact, the components of $y \cdot T m_{k} \cdot c$ are $y \cdot T m_{k} \cdot T V_{i k}=y \cdot T m_{i}=m_{i+1} \cdot d_{i}$, and $m_{i+1} \cdot d_{i}$ are the components of $m_{k} \cdot d$. By the diagonal fill-in, there 


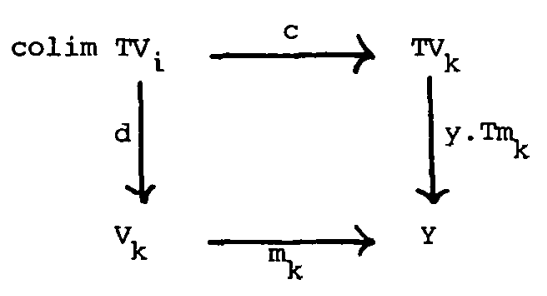

is $b_{k}: T V_{k} \rightarrow V_{k}$ such that

$$
m_{k}:\left(v_{k}, v_{k}\right) \rightarrow(Y, y)
$$

becomes a homomorphism. Since $f$ factors through this monomorphism in $X$. it factors also in $\mathrm{Alg}(T)$ (because monos are initial) and hence, $m_{k}$ is an isomorphism. Thus, $Y$ is isomorphic to an object of $X^{*}$.

In order to study the solidness of the categories of T-algebras, we shall work with the concept of a partial T-algebra as a partial morphism from $T X$ to $X$. Thus, a partial $T$-algebra is represented by a span $T X \stackrel{m}{\longleftarrow} X_{0} \stackrel{x}{\longrightarrow} X$ with $m$ a mono. The category $P$ Alg(T) of partial $T$-algebras has as morphisms from $T X \stackrel{m}{\longleftarrow} X_{0} \stackrel{x}{\longrightarrow} X$ into $T X^{\prime} \stackrel{m^{\prime}}{\longleftarrow} X_{0}^{\prime} \stackrel{x^{\prime}}{\longrightarrow} X^{\prime}$ those $X$-maps $f: X \rightarrow X^{\prime}$ for which there exists a (unique) $f_{0}: X_{0} \rightarrow X_{0}^{\prime}$ such that the following diagram commutes. This category contains A1g(T)

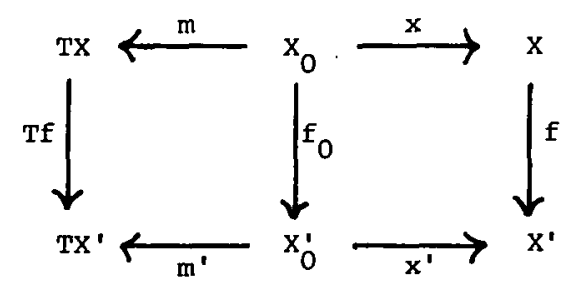

as a full subcategory in the obvious sense.

2.9 PROPOSITION. Let $x$ be a finitely complete category with intersections. Then for each $T: X \rightarrow X$ the category $P$ Alg $(T)$ is monotopological, that is, has initial lifts of stmetured monosources. 
Proof. Let us work with a larger category $R$ Alg(T) of relational algebras, that is, relations from $T X$ to $X$ (represented by a span $T X \stackrel{m}{=} X_{0} \stackrel{x}{\rightarrow} X$ such that $m$ and $x$ are collectively mono) with morphisms defined as above. We prove that $R$ Alg(T) is topological and then we conclude that $P \mathrm{Alg}(T)$ is monotopological as follows : given a structured monosource in $P$ Alg $(T)$ :

$$
X \stackrel{f_{i}}{\longrightarrow}\left(T Y_{i} \stackrel{m_{i}}{\longleftarrow} X_{i} \stackrel{y_{i}}{\longrightarrow} Y_{i}\right) \quad(i \in I)
$$

we can find its initial lift $T X \stackrel{m}{\stackrel{4}{S}} X^{\prime} \stackrel{x}{\longrightarrow} X$ in $R$ Alg(T) and then $m$ is a mono, hence, this is an initial lift in $P$ Alg(T). In fact, let $m \cdot a_{1}=m \cdot a_{2}:$

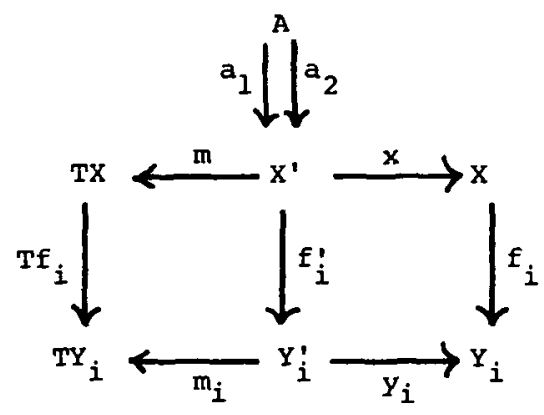

For each $i \in I$ we have $f_{i}^{!} \cdot a_{1}=f_{i}^{!} \cdot a_{2}$ (since $m_{i}$ is a mono, and $\left.m_{i} \cdot f_{i}^{\prime} \cdot a_{1}=m_{i} \cdot f_{i}^{\prime} \cdot a_{2}\right)$ and hence, $f_{i} \cdot\left(x \cdot a_{1}\right)=f_{i} \cdot\left(x \cdot a_{2}\right)$, and since $\left(f_{i}\right)$ is a monosource, we get $x, a_{1}=x \cdot a_{2}$. Since $x$ and $m$ are collectively mono, this proves $a_{1}=a_{2}$.

To prove that $R \dot{A} \mathrm{~g}(T)$ is topological, we use that fact that $X$ has (extremal epi, mono)-factorizations of morphisms. Let a structured sink be given in $R \operatorname{Alg}(T)$ :

$$
\left(T Y_{i} \stackrel{m_{i}}{\stackrel{y_{i}}{\longrightarrow}} Y_{i}\right) \stackrel{f_{i}}{\longrightarrow} X \quad(i \in I) .
$$

Denote by $\bar{m}_{i}: Y_{i}+Y_{i} \times T Y_{i}$ the mono with components $y_{i}$ and $m_{i}$, and 
let us factor $\left(f_{i} \times T f_{i}\right) \cdot \bar{m}_{i}$ as an extremal epi $e_{i}: Y_{i}^{\prime} \rightarrow Y_{i}^{*}$ followed by a mono $m_{i}{ }^{*}: Y_{i}^{*}+X \times T X$. Since $X$ has intersections, the lattice of subobjects of $X \times T X$ is large-complete, and hence, there is a union

$$
m^{*}=\underset{i \in I}{\cup} m_{i}^{*}, m_{i}^{*}=m^{*} \cdot d_{i} \text { for } i \cdot \epsilon I
$$

The mono $m^{*}$ represents a relation $T X \stackrel{m}{\longleftarrow} X^{\prime} \stackrel{x}{\longrightarrow} X$, and we verify that this is the final lift of the given sink in $R$ Alg(T) :

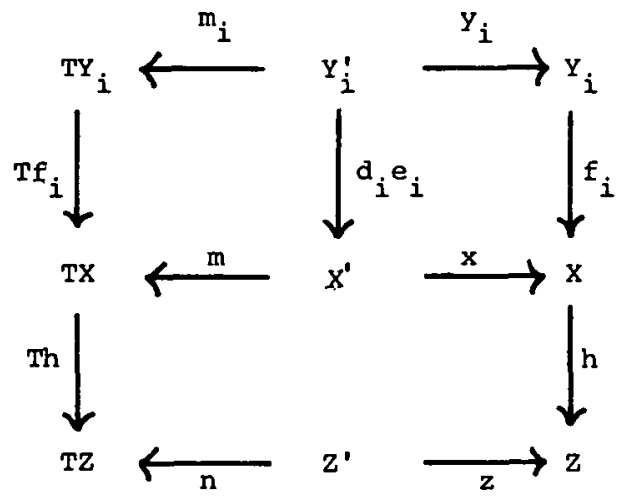

First, $f_{i}:\left(T Y \stackrel{m_{i}}{\longleftarrow} Y_{i}^{\prime} \stackrel{y_{i}}{\longrightarrow} Y\right)+\left(T X \stackrel{m}{\longleftarrow} X^{\prime} \stackrel{x}{\longrightarrow} X\right)$ is a

homomorphism, that is $\left(f_{i} \times T f_{i}\right) \cdot \bar{m}_{i}$ factors through $m^{*}$ : in fact, $\left(f_{i} \times T f_{i}\right) \cdot \bar{m}_{i}=m_{i}{ }^{*} \cdot e_{i}=m^{*} \cdot d_{i} \cdot e_{i}$. Next, let $T Z \stackrel{n}{\longleftarrow} Z^{\prime} \stackrel{z}{\longrightarrow} Z$ be an object and $h: X \rightarrow Z$ a morphism such that each $h \cdot f_{i}(i \in I)$ is a homomorphism. This means that for each $i \in I$,

$$
(h \times T h) \cdot\left(f_{i} \times T f_{i}\right) \cdot \bar{m}_{i} \text { factors through } \bar{n}
$$

where $\bar{n}: Z^{\prime} \rightarrow Z \times T Z$ is the mono with the components $z$ and $n$. Let us form the pullback $(\tilde{n}, \bar{h})$ of $\bar{n}$ and $h \times T h$, see below.

For each $i$, we see that $\left(f_{i} \times T f_{i}\right) \cdot \bar{m}_{i}=m_{i}{ }^{*} \cdot e_{i}$ factors through the mono $\tilde{n}$ and hence, $m_{i}^{*}$ factors through $\tilde{n}$. Therefore, $m^{*}$ factors through $\tilde{n}$, and this proves that $(h \times T h) . m^{*}$ factors through $\bar{n}$. In 


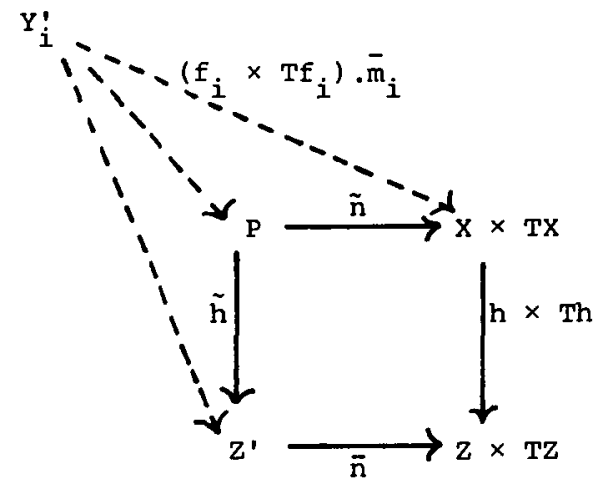

other words, $h:\left(T X \stackrel{m}{\longleftarrow} X^{\prime} \stackrel{x}{\longrightarrow} X\right) \rightarrow\left(T Z \stackrel{n}{\longleftarrow} Z^{\prime} \stackrel{z}{\longrightarrow} Z\right)$ is a homomorphism.

COROLLARY. Let $x$ be complete and wellpowered. Then each $x^{\top}$ and $A Z g(T)$ is a strongly fibre-small category, that is, every $X \in X$ has $a$ small set of representative structured maps from $X$ to algebras.

In fact, since $X$ is wellpowered, $P$ Alg(T) is fibre-small and, being monotopological, it is thus strongly fibresmall. Consequently, each full subcategory is strongly fibresmall.

The equivalence of (ii) and (iii) in the following proposition has been established, under more restrictive hypotheses, in [13].

2.10 PROPOSITION. Let $T=(T, n, \mu)$ be a monad on a finitely complete category $X$ with intersections and coequalizers. Equivalent are:

(i) $x^{\top}$ is solid;

(ii) $X^{\top}$ has coequalizers;

(iii) partial T-algebras have free T-completions, that is, $x^{T}$ is reflective in $P A \lg (T)$.

Proof. $i \rightarrow i i$ is clear since solidness implies lifting of colimits. ii $\rightarrow$ iii For each partial $T$-algebra $T X \stackrel{m}{\longleftarrow} X_{0} \stackrel{x}{\longrightarrow} X$ we form the coequalizer in $x^{\top}$ : 


$$
\left(T X_{0}, \mu\right) \underset{\mu \cdot T m}{\stackrel{T x}{\longrightarrow}}(T X, \mu) \stackrel{c}{\longrightarrow}(Y, y)
$$

Then the following diagram

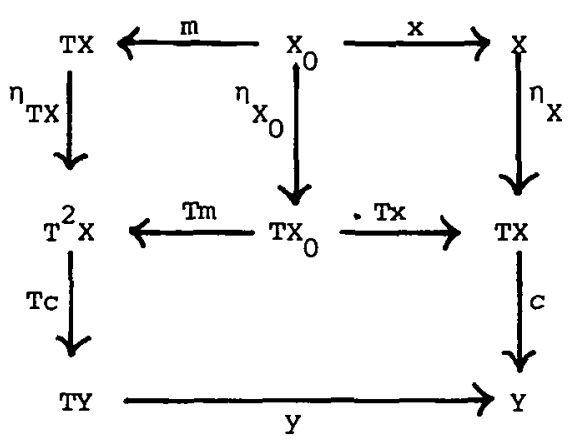

commutes, and since $y \cdot T c=c \cdot \mu_{X}$ implies that $y \cdot T C \cdot \eta_{T X}=y \cdot T c \cdot T \eta_{X}$, we conclude that

$$
c . n_{X}:\left(T X \stackrel{m}{\longleftarrow} X_{0} \stackrel{x}{\longrightarrow} X\right) \rightarrow(Y, y)
$$

is a homomorphism in $P \mathrm{Alg}(T)$. To verify that this is the reflection of the given partial T-algebra, let

$$
h:\left(T X \stackrel{m}{\longleftarrow} X_{0} \stackrel{x}{\longrightarrow} X\right) \rightarrow\left(Y^{\prime}, y^{\prime}\right)
$$

be a homomomorphism in $P$ Alg $(T)$. Then the T-homomorphism $y^{\prime} . T h:(T X, \mu) \rightarrow\left(Y^{\prime}, y^{\prime}\right)$ merges $T x$ and $\mu . T m:$ since $h$ is a homomorphism, that is $h . x=y^{\prime}$. Th.m, we have

$$
\begin{aligned}
\left(y^{\prime} \cdot T h\right) \cdot T x & =y^{\prime} \cdot T(h \cdot x) \\
& =y^{\prime} \cdot T y^{\prime} \cdot T^{2} h \cdot T m \\
& =y^{\prime} \cdot \mu_{Y^{\prime}} \cdot T^{2} h \cdot T m \\
& =\left(y^{\prime} \cdot T h\right) \cdot \mu_{X} \cdot T m .
\end{aligned}
$$

Thus, $y^{\prime}$. Th factors through $c$, and hence, $h$ factors through $c . n_{X}$. $i i i \rightarrow i$ This follows from the preceding proposition. 
COROLLARY. For $X$ as above, $A \mathrm{Zg}(T)$ is solid if and only if $T: X \rightarrow X$ is a varietor such that $A$ Ig $(T)$ has coequalizers.

2.11 EXAMPLE. A concrete category over set which is solid, but is not topologically-algebraic.

Let $X=\Delta$ Pos denote the category of $\Delta$-complete posets, (that is, such that each non-empty up-directed set has a join) and $\Delta$-continuous maps (that is, those preserving directed foins). Let $T: X \rightarrow X$ be the "discretization" functor, assigning to each poset $X$ the discretely ordered poset $T X$ with the same underlying set. Then $A l g(T)$ is the category of ordered unary algebras where the order is $\Delta$-complete (and unrelated to the operation). We shall prove that $A l g(T)$, as a concrete category over set, is solid but not topologically-algebraic.

(a) $\operatorname{Alg}(T)$ is solid over $\triangle$ Pos.

In fact, $A l g(T)$ is reflexive in $P A l g(T)$ : for each partial algebra $(X, \alpha)$ (where $X$ is a $\Delta$-complete poset, and $\alpha: D \rightarrow X$ is a map, $D \subseteq X$ ). the free completion is the following algebra

$$
(X+(X-D) \times \omega, \bar{\alpha})
$$

where $(X-D) \times \omega$ is discretely ordered, $X+(X-D) \times \omega$ is the (cardinal) sum, and

$$
\begin{aligned}
\bar{\alpha}(x) & =\alpha(x) \text { if } x \in D \\
\bar{\alpha}(x) & =(x, 0) \text { if } x \in X-D \\
\bar{\alpha}(x, i) & =(x, i+1) \text { if }(x, i) \in(X-D) \times \omega .
\end{aligned}
$$

(b) $\triangle$ Pos is solid over set.

This follows from [2, Theorem 2E3]: for solidness over set it is sufficient to have concrete products, small fibres, and bounded generation. The first two properties are obvious, for the last one we observe that given a subset $M$ of a $\Delta$-complete poset $X$, then the closure $\bar{M}$ of $X$ under all existing joins in $X$ is a subobject of $X$ with card $\bar{M} \leq 2$ card $M$. 
(c) Alg(T) is not topologically-algebraic over set.

For each infinite ordinal $i$ we denote by $\left(i+1, \gamma_{i}\right)$ the $T$-algebra with the usual ordering on $i+1=\{0,1, \ldots, i\}$ and with $\gamma_{i}(j)=j+1$ for $j<i, \gamma_{i}(i)=i$. We have a source of inclusion maps.

$$
f_{i}:(X, \alpha) \rightarrow\left(i+1, \gamma_{i}\right) \quad(i \in \text { ord, } i \geq \omega)
$$

where $X$ is the discretely ordered set of all natural numbers, and $\alpha$ is the successor function. Let $g_{i}:(Y, \beta)+\left(i+1, \gamma_{i}\right)$ be an initial source through which our source factors $\left(f_{i}=g_{i} . e\right)$ - we shall derive a contradiction by exhibiting, for each ordinal $i$, a strictly increasing chain $y_{0}<y_{1}<\ldots<y_{j}<\ldots(j<i)$ in $y$ with $g_{k}\left(y_{j}\right)=j$ for all $k \geq j$.

$$
\text { First, put } y_{0}=e(0) \text {. We have } g_{k}\left(y_{0}\right)=f_{k}(0)=0
$$

Given such an $i$-chain $y_{0}<y_{1}<\ldots<y_{j}<\ldots$ put

$$
\begin{aligned}
& y_{i}=\underset{j<i}{\vee} y_{j} \text { if } i \text { is a limit ordinal } \\
& y_{i}=\beta\left(y_{i-1}\right) \text { if } i \text { is isolated. }
\end{aligned}
$$

In the first case, we obtain a strictly increasing $(i+1)$-chain, and by the $\Delta$-continuity of $g_{k}$ we have $g_{k}\left(y_{i}\right)=\underset{j<i}{V} g_{k}\left(y_{j}\right)=\underset{j<i}{V} j=i$. In the latter one, we have $g_{k}\left(y_{i}\right)=g_{k} \cdot \beta\left(y_{i-1}\right)=\gamma_{k} \cdot g_{k}\left(y_{i-1}\right)=\gamma_{k}(i-1)=i$. It remains to prove that $y_{i-1}<y_{i}$. First observe that $y_{i-1} \neq y_{i}$ (because if $y_{i-1}=y_{i}$, then $y_{i}$ is a fixpoint of $\beta$ and hence, $g_{k}\left(y_{i}\right)$ is a fixpoint of $r_{k}$, but this is not true for $k>i$ ). Define a $T$-algebra $A=(X+\{0,1, \ldots, i\}, \delta)$ on the coproduct of $X$ and the chain $i+1$ as follows : $\delta$ agrees with $\alpha$ on $X$, and $\delta$ is the successor map on the second summand except that $\delta(i)=0 \epsilon X$. Define $h: X+\{0,1, \ldots, i\} \rightarrow Y$ by $h(n)=\beta^{n}\left(y_{i}\right)$ for $n \in X$ and $h_{j}=y_{j}$ for $j=0,1, \ldots, i$. This map clearly has the property that $g_{k} \cdot h: A \rightarrow\left(k+1, Y_{k}\right)$ are homomorphisms for all $k \geq \omega$. Consequently, 
$h: A \rightarrow(Y, \beta)$ is a homomorphism, and thus, $y_{i-1}=h(i-1) \leq h(i)=y_{i}$.

Remark. A solid category over set which is not topologically algebraic was first exhibited by Börger and Tholen [5].

Open problem. Does there exist a monad on a "reasonable" category $X$ such that the Eilenberg-Moore category is solid, but not topologicallyalgebraic over $X$ ?

\section{References}

[1] J. Adamek, "Colimits of algebras revisited", BuZZ. AustraZ. Math. Soc. 17 (1977), 433-450.

[2] J. Adamek, Theory of mathematical structures, (D. Reidel Publ. Comp., Dordrecht-Boston-Lancaster, 1983).

[3] J. Adámek and V. Koubek, "Are colimits of algebras easy to construct?", J. Algebra 66 (1980), 226-250.

[4] M. Barr, "Coequalizers and free triples", Math. 2. 116 (1970), 307-322.

[5] R. Borger and w. Tholen, "Remarks on topologically-algebraic functors", Cahiers Topologie Geom. Differentielle 20 (1979), 155-177.

[6] R. Borger, W. Tholen, M.B. Wischnewsky and H. Wolff, "Compact and hypercomplete categories, J. Pure Appl. Algebra 21 (1981), 129-144.

[1] H. Herrlich, R. Nakagawa, G.E. Strecker and T. Titcomb, "Equivalence of semi-topological and topologically-algebraic functors", Canad. J. Math. 32 (1980), 34-39.

[8] R. -E. Hoffman, "Semi-identifying lifts and generalization of the duality theorem for topological functors", Math. Nachr. 74 (1976), 295-307.

[9] S.S. Hong, "Categories in which each monosource is initial", Kyungpook Math. J. 15 (1975), 133-139.

[10] Y.H. Hong, Studies on categories of universal topological algebras, (Thesis, McMaster University, 1974). 
[11] J.R. Isbell, "Small subcategories and completeness", Math. Systems Theory 2 (1968), 27-50.

[12] M. Kelly, "A unified treatement of transfinite constructions for free algebras, free monoids, colimits associated sheaves, and so on", Bull. Austral. Math. Soc. 22 (1980), 1-84.

[13] V. Koubek, J. Reitermann, "Categorical constructions of free algebras, colimits and completions of partial algebras, J. Pure App Z. AZgebra 14 (1979), 195-231.

[14] E.G. Manes, Algebraic Theories, (Springer, New York-Heidelberg-Berlin, 1976).

[15] B.A. Rattray, "Adjoints to functors from categories of algebras", Comm. AZgebra 3 (1975), 563-569.

[16] w. Tholen, "Semi-topological functors I", J. Pure Appl. Algebra 15 (1979), 53-73.

[17] v. Trnkova, "Automata in categories", Lect. Notes Comp. Sci. 32 (Springer, 1975).

[18] V. Trnkova, J. Adámek, V. Koubek and J. Reitermann, "Free algebras, input processes and free monads", Comment. Math. Univ. Carolin. 16 (1975), 339-351.

Faculty of Electrical Engineering

FEL CṼUT Suchbátarova 2

Praha 6,

Czechoslovakia 